\title{
Model-independent Redshift Estimation of BL Lac Objects through VHE Observations
}

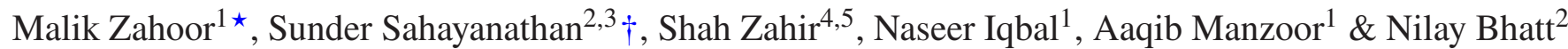 \\ ${ }^{1}$ Department of Physics, University of Kashmir, Srinagar 190006, India. \\ ${ }^{2}$ Astrophysical Sciences Division, Bhabha Atomic Research Center, Mumbai 400085, India. \\ ${ }^{3}$ Homi Bhabha National Institute, Mumbai 400094, India. \\ ${ }^{4}$ Inter-University Center for Astronomy and Astrophysics, Post Bag 4, Ganeshkhind, Pune 411007, India. \\ ${ }^{5}$ Department of Physics, Central University of Kashmir, Ganderbal 191201, India.
}

Accepted XXX. Received YYY; in original form ZZZ

\begin{abstract}
The very high energy (VHE) gamma-ray spectral indices of blazars show strong correlation with the source redshift. Absence of any such correlation in low energy gamma rays and X-rays indicate the presence of Extragalactic Background Light (EBL) induced absorption of VHE gamma rays. By employing a linear regression analysis, this observational feature of blazars is used to constrain the redshift of BL Lac objects which was unknown/uncertain earlier. Additionally, we also compare the observed VHE spectral index-redshift correlation with the ones predicted from commonly adopted EBL models. Our study highlights the deviation of the EBL model based predictions from the observation especially at high redshifts.
\end{abstract}

Key words: Galaxies: distances and redshifts, BL Lacertae objects: general, Cosmology: cosmic background radiation.

\section{INTRODUCTION}

The astral sky in the VHE regime $(E>100 \mathrm{GeV})$ is dominated by blazars, a subclass of radio loud active galactic nuclei (AGN) with a relativistic jet pointing at a small angle with respect to line of sight (Urry \& Padovani 1995). Due to this arrangement, the measured flux over the whole electromagnetic spectrum gets Doppler boosted with apparent variability as short as few minutes (Gaidos, J. A. 1996; Albert et al. 2007; Aharonian et al. 2007d; Arlen et al. 2012). The spectral energy distribution (SED) of blazars is characterised by two broad emission components: the low energy component peaking in the optical-to-X-ray band and the high energy peak located in the gamma-ray bands (Urry \& Padovani 1995). The first component is believed to be the synchrotron emission from a relativistic distribution of electrons in the jet, while the emission process responsible for the second component is still under debate. Under leptonic scenario, the high energy component is explained by inverse Compton (IC) scattering of either the synchrotron (synchrotron self Compton - SSC: (Ginzburg \& Syrovatskii 1965; Tavecchio et al. 1998)) or external (external Compton - EC: (Dermer \& Schlickeiser 1993)) photons by electrons and positrons present in the jet. On the contrary, various hadronic models explain this component through protonsynchrotron emission and/or nuclear cascades (Mücke et al. 2003; Weidinger \& Spanier 2015).

Blazars are further classified as BL Lac objects and flat spectrum radio quasars (FSRQs), with the later exhibiting significant emission and/or absorption lines while such features are absent/weak in the former. Based on the energy at which the synchrotron spectral component peaks $v_{s y}$, blazars are sub-divided into low-frequency peaked

\footnotetext{
^ E-mail: malikzahoor313@gmail.com

$\dagger$ E-mail: sunder@barc.gov.in
}

blazars (LBL, $v_{s y}<10^{14} \mathrm{~Hz}$ ), intermediate-frequency peaked blazars (IBL, $10^{14} \leq v_{s y}<10^{15} \mathrm{~Hz}$ ), high-frequency peaked blazars (HBL, with $10^{15} \leq v_{s y} \leq 10^{17} \mathrm{~Hz}$ ) and extreme high-frequency peaked blazars (EHBL, $v_{s y}>10^{17} \mathrm{~Hz}$ ) (Foffano et al. 2019). While FSRQs are all LBLs, BL Lac objects fall under different categories. Most of the blazars detected by ground based Imaging Atmospheric Cherenkov telescope (IACT) facilities in very high energy gammarays (VHE; $E \geq 100 \mathrm{GeV}$ ) are BL Lac objects belonging to the HBL/EHBL class. EHBLs are relatively less luminous compared to other HBLs and their synchrotron spectral component peaks at energy $>10 \mathrm{keV}$.

Unlike other wavebands, VHE gamma ray emission form blazars undergo significant en route attenuation due to pair production losses with the Extragalactic Background Light (EBL) (Gould \& Schréder 1967). EBL is the diffuse radiation with main contributors as the light from galaxies and the reprocessed dust emission of the universe. Accordingly, the EBL spectrum peaks at UV and IR wavelengths and contain information that trace back to the structure formation epoch of our cosmic evolution (see e.g., Hauser \& Dwek 2001; Dwek \& Krennrich 2013). Direct measurement of EBL is heavily hampered by the presence of strong zodiacal and galactic emission, and hence indirect estimates involving cosmological models are employed. Such estimates broadly fall under two categories namely backward evolution models and forward evolution models. In the former approach, one begins with the existing galaxy population and extrapolates back in time using cosmological models to estimate the EBL (Franceschini \& Rodighiero 2017; Franceschini, A. et al. 2008; Stecker et al. 2006; Pei et al. 1999). The later approach begins with cosmological initial conditions and evolves with time. Here, the model parameters are adjusted to reproduce the observed property of the current universe (Inoue et al. 2013; Gilmore et al. 
2012; Primack 2005). Besides these approaches, some cosmological models use the SED of galaxy stellar population and combine it with the cosmic star formation history to calculate the EBL intensity (Khaire \& Srianand 2015; Kneiske, T. M. \& Dole, H. 2010; Finke et al. 2010). The EBL intensity estimated under these models heavily depends on the adapted cosmological conditions and may vary substantially once the underlying assumptions are relaxed (Dwek \& Krennrich 2013).

The attenuation introduced by the EBL cause the observed VHE spectrum of distant blazars to be significantly different from the source spectrum. In other words, the VHE spectra of blazars carry the signature of EBL and can be used as a probe/test the cosmological EBL models. However, the uncertainty regarding the EBL and the intrinsic VHE spectra of blazars makes the problem self consistent. Broadband spectral modelling of blazars using different emission processes can be used to predict the intrinsic VHE spectra and this in turn can provide constraints on various EBL models (Mankuzhiyil et al. 2010). Nevertheless, the number of free parameters deciding the broadband spectra of blazars are significantly large to be constrained and the ambiguity regarding the nature of the radiating particle is a major hindrance for a realistic estimate of the intrinsic VHE spectrum. Additionally, the detection of the gravitationally lensed blazar S3 $0218+35$ at relatively high redshift of $z=0.944$ (Ahnen, M. L. et al. 2016) poses serious challenges to the existing EBL models and the nature of the intrinsic VHE spectrum of blazars.

The problem can be recast to estimate the distance of $\mathrm{BL}$ Lac objects which lack prominent line emissions (see e.g., Laurent-Muehleisen et al. 1999). Assuming certain EBL model to be valid over large distances and with an acceptable estimate of intrinsic VHE spectrum, an innovative way to estimate source distance can be employed by reproducing the observed VHE spectrum (see e.g., Acciari et al. 2010a; Archer et al. 2018). However, the uncertainty regarding the EBL intensity compounded with the model dependent estimates of the intrinsic VHE spectrum hampers the reliability of this distance estimation, particularly for distant sources. In this work, we propose a novel method to calculate the redshift of the BL Lac objects by exploiting the positive correlation between the observed VHE spectral indices and the source redshifts of blazars for which better estimates of their distance is available. Particularly, this redshift estimate do not depend on the assumptions of EBL model and the intrinsic VHE spectra of blazars. We further extend the study to highlight the discrepancy of four commonly used EBL models at high redshifts.

The paper is organised as follows: In the following section, we perform a detailed correlation study between the spectral index and redshift of blazars followed by the linear regression analysis. In section $\S 3$, we use the regression relations to estimate the redshift of 6 BL Lac objects. The comparison between the predicted VHE spectral index-redshift relation by the EBL models with the observation is presented in section $\S 4$. Finally, the results of the present work are discussed and summarised in section $\S 5$. Throughout this work we adapt a cosmology with $\Omega_{M}=0.3, \Omega_{\Lambda}=0.7$, and $H_{0}=71 \mathrm{kms}^{-1} \mathrm{Mpc}^{-1}$.

\section{EBL SIGNATURE IN VHE SPECTRUM OF BLAZARS}

The presence of EBL induced attenuation in VHE spectra of distant blazars can be readily understood from the positive correlation between the observed VHE indices with redshift of distant blazars. This was first systematically shown by Sinha et al. (2014) considering 29 blazars belonging to HBL and EHBL classes. Absence of significant correlation between the spectral indices in other waveband with the source redshift further asserts this inference. We updated the results of Sinha et al. (2014) by including the recent blazars detected at VHE. This includes additional 3 HBLs and 5 EHBLs. We have also excluded IC 310 from the list of EHBL since there are no substantial evidence to confirm its class. Additionally, we have also included 7 FSRQs in the present study. The sources selected for the present study are listed in $\mathrm{TevCat}^{1}$ which include all the blazars detected by HESS, MAGIC, VERITAS and WHIPPLE. For the sources with more than one observation, we selected the VHE spectral index $(\Gamma)$ corresponding to its lowest flux state. This bias will have only a negligible effect on our study results since the variation in spectral index for an individual source is much less compared to the spectral steepening introduced by EBL (section §5). Nevertheless, since the blazar spectrum is known to be highly variable and to quantify this variation we study the standard deviation in the VHE spectral index of two well observed BL Lac objects Mkn 421 and Mkn 501. For Mkn 421, this was found to be $\approx 0.21$ evaluated independently from the 14 observations by MAGIC and 8 by VERITAS (Aleksi'c, J. et al. 2012; Baloković et al. 2016), while in case of Mkn 501 the standard deviation in VHE spectral index was $\approx 0.14$ estimated from 15 observations by MAGIC and 3 by VERITAS (MAGIC Collaboration: et al. 2020; Aleksi'c, J. et al. 2015). This accounts to $\approx 9 \%$ VHE spectral index variation for Mkn 421 and $\approx 7 \%$ for Mkn 501. We assume similar percentage of variation in the VHE spectral index to be present for all BL Lacs and hence, 9\% additional error is applied along with the observational errors. The number of VHE observations for any FSRQ is too less to repeat a similar treatment and hence we consider only the observational errors for the VHE spectral index. Besides this, we also consider for the present study the average VHE spectral index $\left(\Gamma_{a v}\right)$ estimated from all available VHE observations for HBLs and EHBLs.

The en route absorption of VHE photons by the EBL through pair production process results in the steepening of the observed VHE spectrum of blazars (see e.g., Vassiliev 2000; Mankuzhiyil et al. 2010). This effect will be more pronounced for distant sources than the nearer ones. Hence, one would expect a correlation between the observed VHE spectral indices with the source redshift, provided the sources are assumed to have similar intrinsic VHE spectral indices. To investigate this, we perform Pearson and Spearman rank correlation studies between observed VHE spectral index ${ }^{2}$ and the source redshift for the blazars listed in Table 1 . Since the intrinsic VHE index of HBLs can be harder than FSRQs (Ackermann et al. 2011), we perform the correlation study on individual classes rather than the entire sample. The list of HBLs and FSRQs are given in the top and bottom panel of Table 1. In the middle panel, we provide the list of EHBLs for which the intrinsic VHE spectrum obtained considering various EBL models is extremely hard with an index $<2$ (see e.g., Tavecchio 2014; Tanaka et al. 2014).

\subsection{Spectral index - Redshift Correlation}

To examine the dependence of the observed VHE spectral index $(\Gamma)$ on the redshift $(z)$, we perform a correlation study between these quantities for the ensemble of blazars listed in Table 1. The Spearman rank correlation analysis for the case of HBLs resulted in rank correlation coefficient, $r_{s}=0.63$ with the null hypothesis probability

\footnotetext{
1 http://tevcat.uchicago.edu/

2 We define the spectral index, $\Gamma$, such that $\mathrm{dN} / \mathrm{dE} \propto \mathrm{E}^{-\Gamma}$ (photon $\mathrm{cm}^{-2} \mathrm{~s}^{-1}$ $\left.\mathrm{TeV}^{-1}\right)$.
} 
of $P_{r s}<0.05$. Similarly, the Pearson's correlation analysis resulted in the linear correlation coefficient $\rho=0.72$ with the null hypothesis probability $P_{\rho}<0.05$. In Figure 1 , we show the scatter plot between $\Gamma$ and $z$ for the selected HBLs. For EHBLs the dependence between $\Gamma$ and $z$ is more pronounced with $r_{s}=0.94$ and $P_{r s}<0.05$, while $\rho=0.89$ and $P_{\rho}<0.05$. The scatter plot between $\Gamma$ and $z$ for EHBLs is shown in Figure 2. In case of FSRQs, the correlation is moderate/inconclusive and the results are $r_{s}=0.57$ with $P_{r s}=0.18$ and $\rho=0.69$ with $P_{\rho}=0.087$. The correlation improves to $r_{s}=0.94$ with $P_{r s}=0.004$ and $\rho=0.95$ with $P_{\rho}=0.002$ when the high redshift blazar with spiral morphology, S3 $0218+35(z=0.954)$ is omitted. However, with only 7 FSRQs detected at VHE energies this correlation should be treated only as indicative. The corresponding scatter plot for the FSRQs is given in Figure 3. When the correlation study is repeated with $\Gamma_{a v}$, we found the the results are $r_{s}=0.67$ with $P_{r s}<0.05$ and $\rho=0.75$ with $P_{\rho}<0.05$ for HBLs, and $r_{s}=0.95$ with $P_{r s}<0.05$ and $\rho=0.89$ with $P_{\rho}<0.05$ for EHBLs.

Our study illustrates that there exist a definite correlation between the observed VHE spectral index with the source redshift. This can be attributed to the EBL induced absorption provided the correlation is not associated with the cosmological evolution of blazars. The later presumption can be tested by performing a correlation study between spectral index at lower energies and the redshift. A positive correlation can validate the cosmological evolution or alternatively, falsify the signature of EBL induced absorption on the VHE spectra of distant blazars. We study the correlation between X-ray spectral indices with redshift for HBLs using: 105 months of the Swift-BAT catalog consisting of 16 HBLs (Oh et al. 2018), second ROSAT all-sky survey (2RXS) source catalog containing $34 \mathrm{HBLs}$ (Boller et al. 2016), six years of the Beppo-SAX catalog consisting of 38 HBLs (Donato et al. 2005), and an archival X-ray catalog from ASCA, EXOSAT, Beppo-SAX, ROSAT and EINSTEIN consisting of 61 HBLs (Donato, D. et al. 2001) (Figure 4). The Spearman rank correlation study results are: $r_{s}=-0.15$ with $P_{r s}=0.55$ for Swift-BAT, $r_{s}=0.04$ with $P_{r s}=0.78$ for $2 \mathrm{RXS}, r_{s}=0.02$ with $P_{r s}=0.88$ for Beppo-SAX and $r_{s}=-0.03$ with $P_{r s}=0.79$ for the archival X-ray catalog. Absence of appreciable correlation between the X-ray spectral index and the redshift supports the presence of EBL signature in the VHE spectra of blazars. Additionally, we perform the the Spearman rank correlation between the low-energy gamma-ray $(\mathrm{GeV})$ spectral index and redshift for the 44 HBLs listed in the fourth catalogue of Fermi-LAT (Abdollahi et al. 2020). We obtained $r_{s}=-0.15$ with $P_{r s}=0.85$ suggesting a poor correlation and this further supports the steepening of the VHE spectral index with redshift to be an outcome of EBL induced absorption. Lack of correlation between the spectral index and luminosity in VHE further asserts the correlation between VHE spectral index and redshift is not associated with the Malmquist bais (Sinha et al. 2014).

\subsection{Linear Regression Analysis}

The dependence of the observed VHE spectral index with redshift can be further scrutinized through a linear fit. Such treatments are advantageous in establishing the relation between these two quantities. For HBLs, we found the best fit straight line between $\Gamma$ and $z$ to be

$\Gamma=(5.34 \pm 1.1) z+(2.50 \pm 0.12)$

with $\chi^{2} /$ d.o.f $=12.97 / 22$ and in case of $\Gamma_{a v}$ and $z$

$\Gamma_{a v}=(5.97 \pm 1.15) z+(2.55 \pm 0.13)$

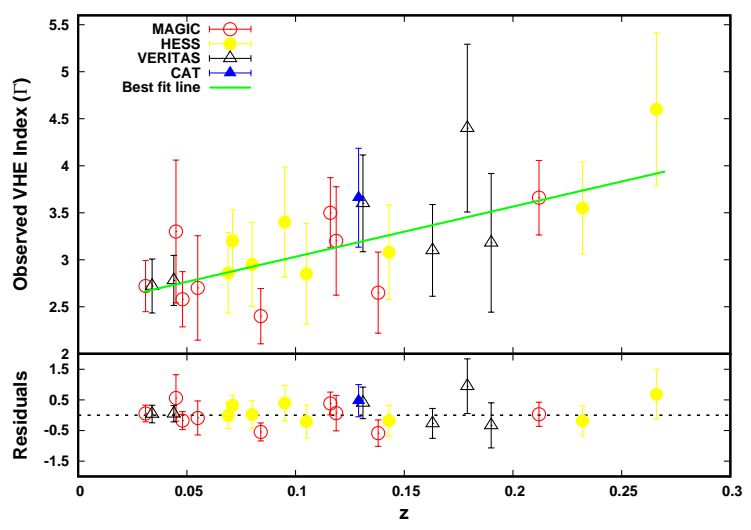

Figure 1. Top: Scatter plot between observed VHE spectral index of the selected HBLs with redshift. Different color/symbol depict observations from different VHE telescopes as mentioned in labels. The solid line (green) is the best-fit straight line to the HBLs with $\chi^{2} /$ d.o.f $=12.97 / 22$, Bottom : Residual plot.

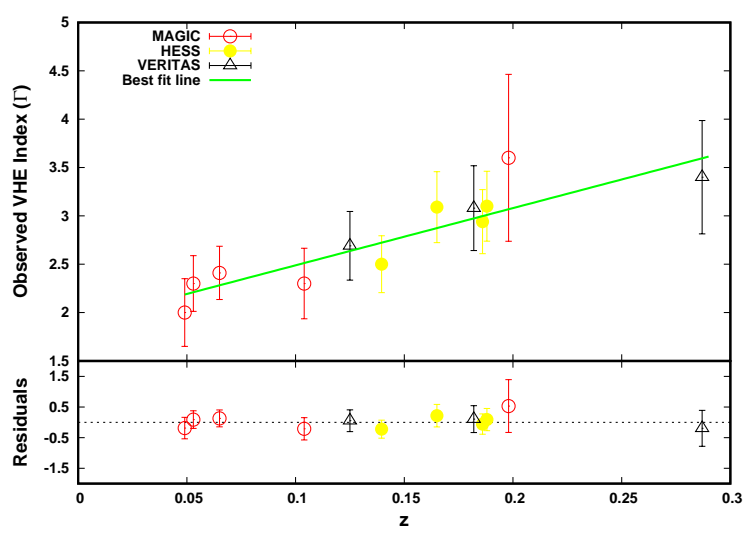

Figure 2. Top: Scatter plot between observed VHE spectral index of the selected EHBLs with redshift. Different color/symbol depict observations from different VHE telescopes as mentioned in labels. The solid line (green) is the best-fit straight line to the EHBLs with $\chi^{2} /$ d.o.f $=2.52 / 10$, Bottom: Residual plot.

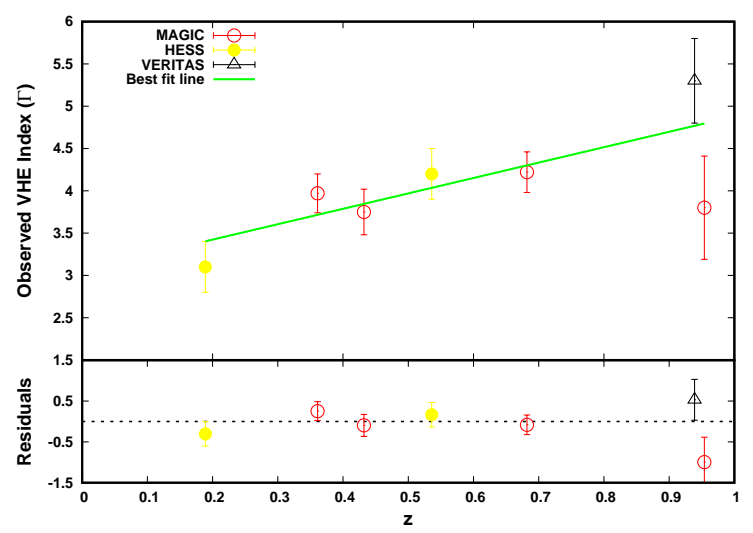

Figure 3. Top: Scatter plot between observed VHE spectral index of the FS$\mathrm{RQs}$ with redshift. Different color/symbol depict observations from different VHE telescopes as mentioned in labels. The solid line (green) is the best-fit straight line to the FSRQs with $\chi^{2} /$ d.o.f $=6.57 / 5$, Bottom: Residual plot. 


\begin{tabular}{|c|c|c|c|c|c|c|c|}
\hline Source Name & Type & $\mathrm{z}$ & $\Gamma$ & $\mathrm{E}_{V H E}$ & Total no. of VHE obs & $\Gamma_{a v}$ & Ref \\
\hline Markarian 421 & HBL & 0.031 & $2.72 \pm 0.12$ & $>0.2$ & 22 & $2.74 \pm 0.56$ & Aleksi'c, J. et al. (2012) \\
\hline Markarian 501 & HBL & 0.034 & $2.72 \pm 0.15$ & $>0.1$ & 18 & $2.30 \pm 0.51$ & Acciari et al. (2011a) \\
\hline 1ES $2344+514$ & HBL & 0.044 & $2.78 \pm 0.09$ & $0.39-8.3$ & 05 & $2.65 \pm 0.27$ & Acciari et al. (2011b) \\
\hline PKS 2155-304 & HBL & 0.116 & $3.5 \pm 0.2$ & $>0.4$ & 02 & $3.51 \pm 0.20$ & Aleksic et al. (2012) \\
\hline 1ES $1959+650$ & HBL & 0.048 & $2.58 \pm 0.18$ & $0.18-2$ & 04 & $2.82 \pm 0.31$ & Tagliaferri et al. (2008) \\
\hline H $1426+428$ & HBL & 0.129 & $3.66 \pm 0.41$ & $>0.25$ & 03 & $3.25 \pm 0.80$ & Djannati-Ataï, A. et al. (2002) \\
\hline PKS 2005-489 & HBL & 0.071 & $3.20 \pm 0.16$ & $0.3-5$ & 02 & $3.60 \pm 0.43$ & Acero et al. (2010) \\
\hline Markarian 180 & HBL & 0.045 & $3.3 \pm 0.7$ & $>0.25$ & 01 & $\ldots$ & Albert et al. (2006) \\
\hline PKS 0548-322 & HBL & 0.069 & $2.86 \pm 0.34$ & $>0.25$ & 02 & $2.83 \pm 0.45$ & Aharonian et al. (2010) \\
\hline 1ES $1011+496$ & HBL & 0.212 & $3.66 \pm 0.22$ & $>0.15$ & 04 & $3.49 \pm 0.74$ & Aleksić et al. (2016) \\
\hline RGB J0152+017 & HBL & 0.08 & $2.95 \pm 0.36$ & $>0.3$ & 01 & $\ldots$ & Aharonian et al. (2008b) \\
\hline 1ES $0806+524$ & HBL & 0.138 & $2.65 \pm 0.36$ & $>0.3$ & 02 & $3.12 \pm 1.06$ & Aleksić et al. (2015) \\
\hline RBS 0413 & HBL & 0.19 & $3.18 \pm 0.68$ & $>0.25$ & 03 & $3.19 \pm 0.97$ & Aliu et al. (2012a) \\
\hline 1ES $1440+122$ & HBL & 0.163 & $3.1 \pm 0.4$ & $0.2-1.3$ & 02 & $3.25 \pm 0.80$ & Archambault et al. (2016) \\
\hline RX J0648+1516 & HBL & 0.179 & $4.4 \pm 0.80$ & $>0.2$ & 01 & $\ldots$ & Aliu et al. (2011) \\
\hline B3 $2247+381$ & HBL & 0.118 & $3.2 \pm 0.5$ & $>0.2$ & 01 & $\ldots$ & Aleksić et al. (2012) \\
\hline SHBL J001355.9-185406 & HBL & 0.095 & $3.4 \pm 0.5$ & $>0.31$ & 01 & $\ldots$ & Abramowski et al. (2013b) \\
\hline 1RXS J101015.9-311909 & HBL & 0.143 & $3.08 \pm 0.42$ & $>0.2$ & 02 & $3.09 \pm 0.65$ & Abramowski et al. (2012) \\
\hline 1ES 1312-423 & HBL & 0.105 & $2.85 \pm 0.47$ & $>0.28$ & 01 & $\ldots$ & Abramowski et al. (2013a) \\
\hline 1ES $1215+303$ & HBL & 0.131 & $3.6 \pm 0.4$ & $>0.2$ & 03 & $3.38 \pm 0.65$ & Aliu et al. (2013) \\
\hline 1ES $1741+196$ & HBL & 0.084 & $2.4 \pm 0.2$ & $0.08-3$ & 02 & $2.55 \pm 0.72$ & Ahnen et al. (2017) \\
\hline 1ES $1727+502$ & HBL & 0.055 & $2.7 \pm 0.5$ & $>0.15$ & 03 & $2.40 \pm 0.58$ & Aleksić et al. (2014) \\
\hline PKS 0301-243 & HBL & 0.266 & $4.6 \pm 0.7$ & $>0.2$ & 01 & $\ldots$ & Abramowski et al. (2013c) \\
\hline 1RXS J023832.6-311658 & HBL & 0.232 & $3.55 \pm 0.371$ & $>0.1$ & 01 & $\ldots$ & Gaté et al. (2017) \\
\hline 1ES 1101-232 & EHBL & 0.186 & $2.94 \pm 0.20$ & $>0.22$ & 01 & $\ldots$ & Aharonian et al. (2007a) \\
\hline Н 2356-309 & EHBL & 0.165 & $3.09 \pm 0.24$ & $0.2-1.3$ & 02 & $3.07 \pm 0.28$ & Aharonian et al. (2006b) \\
\hline 1ES $1218+304$ & EHBL & 0.182 & $3.08 \pm 0.34$ & $0.16-1.8$ & 02 & $3.04 \pm 0.52$ & Fortin et al. (2008) \\
\hline $1 \mathrm{ES} 0229+200$ & EHBL & 0.1396 & $2.50 \pm 0.19$ & $0.5-15$ & 02 & $2.46 \pm 0.21$ & Aharonian et al. (2007c) \\
\hline 1ES 0347-121 & EHBL & 0.188 & $3.10 \pm 0.23$ & $0.25-3$ & 01 & $\ldots$ & Aharonian et al. (2007b) \\
\hline RGB J0710+591 & EHBL & 0.125 & $2.69 \pm 0.26$ & $>0.3$ & 01 & $\ldots$ & Acciari et al. (2010b) \\
\hline 1ES 0414+009 & EHBL & 0.287 & $3.4 \pm 0.5$ & $0.23-0.85$ & 03 & $3.42 \pm 0.62$ & Aliu et al. (2012b) \\
\hline RBS 0723 & EHBL & 0.198 & $3.60 \pm 0.80$ & $>0.1$ & 01 & $\ldots$ & Acciari et al. (2020a) \\
\hline $1 \mathrm{ES} 2037+521$ & EHBL & 0.053 & $2.30 \pm 0.2$ & $>0.1$ & 01 & $\ldots$ & Acciari et al. (2020a) \\
\hline PGC 2402248 & EHBL & 0.065 & $2.41 \pm 0.17$ & $>0.22$ & 01 & $\ldots$ & Acciari et al. (2019) \\
\hline TXS $0210+515$ & EHBL & 0.049 & $2.0 \pm 0.30$ & $>0.1$ & 02 & $1.95 \pm 0.41$ & Acciari et al. (2020a) \\
\hline RGB J2042+244 & EHBL & 0.104 & $2.3 \pm 0.30$ & $>0.1$ & 01 & $\ldots$ & Acciari et al. (2020a) \\
\hline $3 \mathrm{C} 279$ & FSRQ & 0.536 & $4.20 \pm 0.30$ & $>0.1$ & $\ldots$ & $\ldots$ & H. E. S. S. Collaboration et al. (2019) \\
\hline PKS 1510-089 & FSRQ & 0.361 & $3.97 \pm 0.23$ & $>0.15$ & $\ldots$ & $\ldots$ & MAGIC Collaboration et al. (2018) \\
\hline $4 C+21.35$ & FSRQ & 0.432 & $3.75 \pm 0.27$ & $0.07-0.4$ & $\ldots$ & $\ldots$ & Aleksić et al. (2011b) \\
\hline S3 $0218+35$ & FSRQ & 0.954 & $3.80 \pm 0.61$ & $0.065-0.175$ & $\ldots$ & $\ldots$ & Ahnen et al. (2016b) \\
\hline PKS $1441+25$ & FSRQ & 0.939 & $5.3 \pm 0.5$ & $0.08-0.2$ & $\ldots$ & $\ldots$ & Abeysekara et al. (2015) \\
\hline PKS $0736+017$ & FSRQ & 0.189 & $3.1 \pm 0.30$ & $>0.1$ & $\ldots$ & $\ldots$ & H. E. S. S. Collaboration et al. (2020) \\
\hline B2 $1420+32$ & FSRQ & 0.682 & $4.22 \pm 0.24$ & $>0.1$ & $\ldots$ & $\ldots$ & Acciari et al. (2020b) \\
\hline
\end{tabular}

Table 1. List of BL Lac objects detected in VHE used in this work.Top group lists the HBL's, Middle group lists the extreme HBL's and bottom group lists the FSRQs. Column description, 1: Source Name 2: Source classification 3: Redshift (z) 4: Observed VHE Index (Г) during its lowest flux state 5: VHE spectral range $(\mathrm{TeV})$ 6: Total number of VHE observations available in literature (http://tevcat.uchicago.edu/) 7: Average observed VHE index considering all available observations (with propagated errors) 7: References of the Low VHE flux state.

with $\chi^{2} /$ d.o.f $=10.99 / 22$. In Figure 1 , we show the best fit line for the case of $\Gamma$ and $z$ as solid green line with the residuals in the bottom panel. The residuals are evenly spread over the source redshifts and this advises that a straight line is capable of explaining the observed trend. We also produced a normal quantile plot to identify the distribution of residuals. This also favours a normal distribution of residuals and hence the regression relations given by equation 1 \& 2 can be used for prediction.

The analysis is repeated for the case of EHBLs and FSRQs. For EHBLs, the linear regression analysis results are

$\Gamma=(5.92 \pm 0.9) z+(1.90 \pm 0.12)$

with $\chi^{2} /$ d.o.f $=2.52 / 10$ and

$\Gamma_{a v}=(5.51 \pm 0.88) z+(1.95 \pm 0.12)$ with $\chi^{2} /$ d.o.f $=4.41 / 10$. In Figure 2 we show the best fit line with the residuals in the bottom panel for $\Gamma$ and $z$. The low value of $\chi^{2} /$ d.o.f results from the large errors in the data; however, a straight line is the simplest function that can be fitted and the fit statistics can be improved with a larger sample and precise index measurements. For FSRQs, the linear regression analysis results are

$\Gamma=(1.82 \pm 0.62) z+(3.06 \pm 0.33)$

with $\chi^{2} /$ d.o.f $=6.57 / 5$ and the best fit line is shown in Figure 3 .

\section{REDSHIFT ESTIMATION}

The BL Lac class of blazars often lack emission/absorption line features and this makes it almost impossible to identify the source 


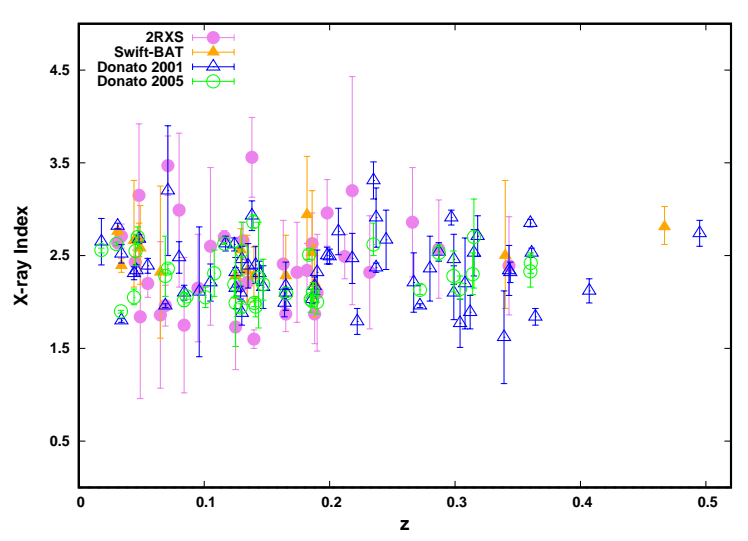

Figure 4. Scatter plot between observed X-ray spectral index of HBLs with redshift. The filled violet circles are from the ROSAT catalog (Boller et al. 2016), the yellow filled triangles from the Swift-BAT catalog (Oh et al. 2018)), the blue triangles from archival X-ray catalog (Donato, D. et al. 2001), and the green circles from the Beppo-SAX catalog (Donato et al. 2005).

redshift through optical spectroscopy. Alternatively, the steepening of the VHE spectrum due to the EBL can be used to estimate the source redshift/distance. Usual methods involve prediction of intrinsic VHE spectrum at source through theoretical modelling and assumption of certain EBL model (see e.g., Mankuzhiyil et al. 2010). Instead, the regression relations given in section $\$ 2$ can also be used to estimate the redshift of blazars. The advantage is the estimated redshifts do not depend either on blazar SED model or the EBL models rather it relies only on the observed correlation between $\Gamma$ and $z$. Below we use the regression relations (equation 1,2,3 and 4) to estimate the redshift of five HBLs and one EHBL for which this information is uncertain. The estimated redshifts along with earlier estimates are given in Table 2 .

\section{ES 2322-409}

The BL Lac object 1ES 2322-409 was first detected in VHE by HESS during 2004 (Abdalla et al. 2018). A tentative redshift of $0.17359 \pm$ 0.00018 for 1ES 2322-409 was first suggested from the combined redshift and peculiar velocity survey over the southern sky $\left(|\mathrm{b}|>10^{\circ}\right)$, namely 6DF Galaxy survey (Jones et al. 2009a). This estimation is based upon low signal-to-noise ratio spectrum and the evidence for the absorption line is weak (Abdalla et al. 2018). On the other hand, if we consider the redshifts of the galaxies obtained from shallow surveys (Jones et al. 2009a; Vettolani, G. et al. 1998; Shectman et al. 1996; Ratcliffe et al. 1996) which are located close to 1ES 2322409 , one would estimate the redshift as 0.06 . However, it lacks the justification that this source can be a part of the selected group of galaxies.

The VHE observations of 1ES 2322-409 was reported by (Abdalla et al. 2018) and the spectrum can be well described by a power law with an index $\Gamma=3.40 \pm 0.66$ (stat) \pm 0.20 (sys). The photon index falls well within the range of indices used for the correlation study of HBLs (Figure 1) and hence the regression relations (equation 1 and 2) can be used to estimate the redshift of 1ES 2322409. Using these relations we are able to constrain the source redshift as $0.17 \pm 0.13$ and $0.14 \pm 0.12$ respectively. Unfortunately, the large error bar in the spectral index reflect as significant uncertainty on the estimated redshift ${ }^{3}$.

${ }^{3}$ Given $m$ and $c$ are the slope and y-intercept of the best fit regression line,

\section{H $1722+119$}

H 1722+119 was identified as a BL Lac object independently by Griffiths et al. (1989) and Brissenden et al. (1990), and its optical spectrum is largely featureless. An absorption feature was initially reported by Aleksić et al. (2011a) and estimated the source redshift as 0.018; nevertheless, this was not confirmed by other optical observations (Veron-Cetty \& Veron 1993; Falomo et al. 1993, 1994). Interestingly, observations of the source using ESO's Very Large Telescope did not show any features in its optical spectrum (Sbarufatti et al. 2006) and the authors derived a lower limit of redshift as $z>0.17$. Later using the spectrograph X-shooter of ESO's Very Large Telescope the lower limit of redshift was modified as $z>0.35$ (Landoni, M. et al. 2014). Using Nordic Optical Telescope and following the technique described by Sbarufatti et al. (2005), Farina et al. (2012) obtained the redshift lower limit as $z>0.4$. A redshift estimate of $\mathrm{z}=0.34 \pm 0.15$ using VHE observations by MAGIC was put forth by (Ahnen et al. 2016a). The authors used the fact that the photon index at VHE cannot be harder than the one measured at high energy by Fermi. The redshift estimation was done by considering a EBL model by Franceschini, A. et al. (2008).

For the estimation of redshift for $\mathrm{H} 1722+119$, we use the spectral information obtained from the MAGIC observations during 17-22 May 2013 for six consecutive nights (Ahnen et al. 2016a). The VHE spectrum can be well fitted by a power-law with photon index $\Gamma=3.3$ \pm 0.3 (stat) \pm 0.2 (sys). This index corresponds to a redshift of 0.15 \pm 0.07 using equation 1 and $0.13 \pm 0.06$ using equation 2 . These values are much less than the redshift lower limits obtained from the featureless optical spectra. Though the reasons for this deviation are not very clear, probably the intrinsic spectrum is dominated by the non-thermal emission from the jet and hence the source may lack optical line features. The earlier estimate based on VHE study involves extrapolation of the contemporaneous low energy gammaray spectrum to VHE and this may be the reason for the difference.

\section{PKS 1440-389}

PKS 1440-389 is one of the bright blazars in the Fermi energy range and has been classified as high energy synchrotron peaked blazar by the 3rd Fermi AGN Catalog (Ackermann et al. 2015). Based on the 6dF Galaxy Survey (Jones et al. 2004) a redshift of 0.065 was suggested for the blazar PKS 1440-389. However, due to poor spectral quality this estimate was not included in the $6 \mathrm{dF}$ catalog (Jones et al. 2009b). Under photo-hadronic interpretation of VHE emission and using EBL model by Franceschini, A. et al. (2008), Sahu et al. (2019) constrained the redshift of PKS 1440-389 in the range $0.14 \leq \mathrm{z} \leq 0.24$. Extrapolating the high energy spectrum observed by Fermi to VHE energies and using the EBL model by Domínguez et al. (2010), Abdalla et al. (2020a) estimated the upper limit of redshift as $z<0.53$. In spite of all these measurements at different wavelength, the redshift of PKS 1440-389 is still uncertain with the best limit being $0.14<\mathrm{z}<2.2$ (Shaw et al. 2013).

We considered the VHE spectra index of PKS 1440-389 from two spectral studies by HESS during Feb 2012 (Prokoph et al. 2015; Abdalla et al. 2020b). The mean spectral index from these two observations is $3.66 \pm 0.39$. Using the regression relations (equation 1

the error on redshift, $z=(\Gamma-c) / m$, is estimated as

$$
\Delta z=z \sqrt{\left(\frac{\Delta \Gamma}{\Gamma-c}\right)^{2}+\left(\frac{\Delta c}{\Gamma-c}\right)^{2}+\left(\frac{\Delta m}{m}\right)^{2}} .
$$


and 2) this can be translated into the source redshift as $0.21 \pm 0.09$ and $0.19 \pm 0.08$. These redshift estimates are comparable with earlier values.

\section{PKS 1424+240}

PKS $1424+240$ is a BL Lac object with a hard high energy spectrum (Abdo et al. 2009). The source was first detected at VHE by VERITAS (Acciari et al. 2010a) and later by MAGIC (Aleksi'c, J. et al. 2014). Photometric redshift obtained through spectral fitting of optical/UV data provides an upper limit as $<1.11$ (Acciari et al. 2010a). A lower limit $>0.6$ can be inferred from the $\mathrm{Ly}_{\alpha}$ and $\mathrm{Ly}_{\beta}$ absorption features in far UV spectra (Furniss et al. 2013). Extrapolating the high energy spectra to VHE energies and considering different EBL models, Acciari et al. (2010a) suggested a redshift upper limit as $<0.66$. Prandini et al. (2011) adapted a statistical approach to estimate the redshift of PKS 1424+240 using the high energy and VHE spectral index for an ensemble of blazars with known redshifts. Their study suggested the redshift of PKS $1424+240$ as 0.24 .

For the redshift estimation, we used the two VERITAS studies of the source using the VHE observations taken from 2009 to 2011 (Acciari et al. 2010a; Benbow \& VERITAS Collaboration 2015). The photon spectra can be explained well by a power law and the mean photon index of $4.0 \pm 0.58$ has been calculated. Using the mean index and the regression relations (equation 1 and 2), we estimated the source redshift as $0.28 \pm 0.13$ and $0.24 \pm 0.11$. These are well within the limiting values of redshift and also closely agrees with the estimate of Prandini et al. (2011).

\section{PG 1553+113}

PG $1553+113$ is one of the brightest sources known from $\mathrm{X}$ ray to VHE regime located in the Serpens Caput constellation (Costamante \& Ghisellini 2002). The optical spectrum of the source is featureless and this makes the redshift estimation difficult through spectroscopic studies. Constraints derived from the apparent magnitude of host galaxy sets a lower limit on redshift as $\geq 0.25$ (Treves et al. 2007). More stringent estimate on redshift, $0.43<\mathrm{z}<$ 0.58 , is obtained through the study of far-UV absorption line features arising from the interstellar and intergalactic medium (Danforth et al. 2010). Using HESS and MAGIC observations and assuming the intrinsic photon index cannot be harder than 1.5 along with the EBL model proposed by Kneiske et al. (2004), Mazin \& Goebel (2007) derive an upper limit on the redshift as $<0.69$. Sanchez, D. A. et al. (2013) estimated an upper limit on redshift as $<0.64$ from the measured spectral break between the $\mathrm{GeV}-\mathrm{TeV}$ spectral indices. Assuming the intrinsic VHE spectrum cannot be harder than the high energy and using a Bayesian approach, Abramowski et al. (2015a) found the most probable redshift as $0.49 \pm 0.04$.

We used the VHE spectral information of the source from five distinct observations by HESS, MAGIC and VERITAS experiments (Abramowski et al. 2015b; Aliu et al. 2015; Benbow 2011; Aharonian et al. 2008a; Albert et al. 2009). In all the cases the spectrum is well fitted by a power law and the mean index calculated from these observations is $4.51 \pm 0.26$. This corresponds to an redshift of $0.38 \pm 0.10$ and $0.33 \pm 0.08$ estimated from the regression relations (equation 1 and 2) respectively.

\section{HESS J1943+213}

The VHE source HESS J1943+213 is identified as EHBL from HESS observations (Abramowski et al. 2011). A lower limit on the redshift $\mathrm{z}>0.03$ is estimated from the optical spectroscopic studies (Peter, D. et al. 2014); however, this is not a stringent condition since the host galaxy was not well defined. Alternatively, from the expected host galaxy flux of a typical BL Lac object, a lower limit on redshift can be obtained as $\mathrm{z}>0.14$ (Cerruti 2011). Extrapolating the high energy spectrum observed by Fermi to VHE energies and using the EBL model by Franceschini, A. et al. (2008), an upper limit on redshift is obtained as z $<0.45$ (Peter, D. et al. 2014). Similarly, using Fermi and VERITAS observations along with the EBL model by Franceschini, A. et al. (2008), Archer et al. (2018) derived a conservative limit on redshift as $<0.23$. A photo-hadronic interpretation of VHE emission placed the limits on redshift as 0.14 $\leq \mathrm{z} \leq 0.19$ (Sahu et al. 2019). The VHE spectral information of HESS J1943+213 is obtained from HESS observations during 2009 (Abramowski et al. 2011). The spectrum in the energy range 470 $\mathrm{GeV}$ to $6 \mathrm{TeV}$ is well described by a power law with photon index of $3.1 \pm 0.3$. Since the source is classified as EHBL, we use the regression relations (equation 3 and 4) for EHBLs and the constraint on redshift can be obtained as $0.20 \pm 0.06$ and $0.21 \pm 0.06$.

\section{EBL MODEL COMPARISON}

Besides estimation of redshifts, the observed $\Gamma-z$ correlation can also be used to compare the EBL models. Since direct measurement of EBL is not possible, it is estimated using cosmological models. The techniques employed can be broadly categorised as forward evolution models, backward evolution models and some alternate approaches based on the combination of stellar population with cosmic star formation history (section $\S 1$ ). To compare whether the cosmological EBL models can explain the observed $\Gamma-z$ correlation, we select four EBL models by Inoue et al. (2013), Gilmore et al. (2012), Domínguez et al. (2010) and Franceschini, A. et al. (2008), which are widely used for VHE spectral study of blazars. Among these models, Inoue et al. (2013) and Gilmore et al. (2012) are forward evolution models while, Franceschini, A. et al. (2008) is a backward evolution model. The EBL model by Domínguez et al. (2010) differ from these two approaches where it is estimated using the observed evolution of galaxy population over different range of redshifts.

The comparison between these models and the observations is performed by predicting the $\Gamma-z$ dependence due to these models. The observed VHE flux $F_{o}$ at energy $E$ from a blazar is related to its intrinsic source flux $F_{i}$ as

$F_{o}(E)=F_{i}(E, z) e^{-\tau(E, z)}$

where, $\tau$ is the optical depth due to EBL absorption of VHE photons. If we assume the intrinsic source spectrum to be a power law with index $\Gamma_{i}$, then the observed spectral slope $\Gamma_{o}$ at energy $E_{*}$ will be

$\Gamma_{o}\left(E_{*}, z\right)=\Gamma_{i}+\left.\frac{d \tau(E, z)}{d \ln E}\right|_{E_{*}}$

The $\tau(E, z)$ for a given $z$ and $E_{*}$ is estimated using two dimensional linear interpolation of the tabular EBL models and the differentiation is performed numerically. For HBLs and EHBLs, $E_{*}$ is chosen as 1 $\mathrm{TeV}$ while it is $300 \mathrm{GeV}$ for FSRQs. This energy is approximately the mean of the observed energies for the sample chosen in this work. The intrinsic VHE spectral index $\Gamma_{i}$ is obtained by extrapolating the regression relations (equation 1,3 and 5) to $z=0$ and the evolution of $\Gamma_{o}$ is studied against $z$. In Figures 5 and 6, we show the 


\begin{tabular}{|c|c|c|c|c|c|}
\hline \multirow{2}{*}{ Source Name } & \multirow{2}{*}{ Source Type } & \multicolumn{2}{|c|}{ Redshift Estimate (This Work) } & \multicolumn{2}{|c|}{ Previous Estimates } \\
\hline & & $\Gamma$ & $\Gamma_{a v}$ & Optical based study & Model based VHE study \\
\hline 1ES 2322-409 & HBL & $0.17 \pm 0.13$ & $0.14 \pm 0.12$ & $\begin{array}{c}0.17359 \pm 0.00018(\text { Jones et al. } 2009 \mathrm{a}) \\
0.06(\text { Shallow surveys })\end{array}$ & $\ldots$ \\
\hline H $1722+119$ & HBL & $0.15 \pm 0.07$ & $0.13 \pm 0.06$ & $\begin{array}{c}0.018 \text { (Griffiths et al. 1989) } \\
>0.17 \text { (Sbarufatti et al. 2006) } \\
>0.35 \text { (Landoni, M. et al. 2014) } \\
>0.4 \text { (Farina et al. 2012) }\end{array}$ & $0.34 \pm 0.15$ (Ahnen et al. 2016a) \\
\hline PKS 1440-389 & HBL & $0.21 \pm 0.09$ & $0.19 \pm 0.08$ & $\begin{array}{c}0.065 \text { (Jones et al. 2004) } \\
0.14 \leq \mathrm{z} \leq 0.24 \text { (Sahu et al. 2019) } \\
0.14<\mathrm{z}<2.2(\text { Shaw et al. } 2013)\end{array}$ & $<0.53$ (Abdalla et al. 2020a) \\
\hline PKS $1424+240$ & HBL & $0.28 \pm 0.13$ & $0.24 \pm 0.11$ & $\begin{array}{l}<1.11 \text { (Rau, A. et al. 2012) } \\
>0.6 \text { (Furniss et al. 2013) }\end{array}$ & $\begin{array}{l}\approx 0.24 \text { (Prandini et al. 2011) } \\
<0.66 \text { (Acciari et al. 2010a) }\end{array}$ \\
\hline PG $1553+113$ & HBL & $0.38 \pm 0.10$ & $0.33 \pm 0.08$ & $\begin{array}{c}\geq 0.25 \text { (Treves et al. 2007) } \\
0.43<\mathrm{z}<0.58 \text { (Danforth et al. 2010) } \\
0.49 \pm 0.04 \text { (Abramowski et al. 2015a) }\end{array}$ & $\begin{aligned}<0.69(\text { Mazin \& Goebel 2007) } \\
<0.64(\text { Sanchez, D. A. et al. 2013) }\end{aligned}$ \\
\hline HESS J1943+213 & EHBL & $0.20 \pm 0.06$ & $0.21 \pm 0.07$ & $\begin{array}{c}>0.03(\text { Peter, D. et al. 2014) } \\
0.14 \leq \mathrm{z} \leq 0.19(\text { Sahu et al. } 2019) \\
>0.14(\text { Cerruti } 2011)\end{array}$ & $\begin{array}{l}<0.45 \text { (Peter, D. et al. 2014) } \\
<0.23 \text { (Archer et al. 2018) }\end{array}$ \\
\hline
\end{tabular}

Table 2. Redshift identification of 6 BL Lac objects along with the previous estimates. Column description, 1: Source Name 2: Source classification 3: Redshift values estimated in this work using the regression relation between (i) $z$ and $\Gamma$, and (ii) $z$ and $\Gamma_{a v} 4$ : Previous redshift estimations based on the (i) optical study and (ii) VHE study along with references.

predicted $\Gamma_{o}$ corresponding to the different EBL models for the case of HBLs and EHBLs. The prediction closely satisfy the regression line at small redshifts; however, it starts deviating considerably when the redshift approaches 0.3. Since FSRQs are detected at higher redshifts compared to HBLs and EHBLs, they are the better sample to perform this study at large distances. In Figure 7, we show the predicted $\Gamma_{o}$ corresponding to the different EBL models, for the case of FSRQs, which diverge from the regression line significantly ${ }^{4}$. Even though the number of FSRQs used for this study is less, this result highlights the discrepancy of the EBL models in predicting the intrinsic spectrum of high redshift sources. The gray band around the regression lines in Figures 5, 6 and 7 denote the 1- $\sigma$ error band.

\section{DISCUSSION AND SUMMARY}

The observed positive correlation between the observed VHE spectral index of blazars with redshift is interpreted as a result of EBL induced absorption effects. Absence of such correlation in low energy bands further strengthen this inference. This led us to develop a novel technique to estimate the redshift of distant blazars whose optical spectrum is featureless. This method neither depend upon the choice of the EBL spectrum nor the intrinsic source VHE spectrum and hence can be treated as model independent/unbiased. However, blazar spectral indices are observed to vary significantly during different flux states and this can question the effectiveness of the estimated redshifts. Our study involving 14 MAGIC and 8 VERITAS observations of Mkn 421 suggests the standard deviation in the VHE spectral indices to be 0.21 and in case of Mkn 501 it is 0.14 obtained using 15 MAGIC and 3 VERITAS observations.

\footnotetext{
${ }^{4}$ It should be noted that the VHE energy at which FSRQs are detected is relatively less compared to HBLs/EHBLs. Accordingly the target EBL photon energy probed will also be different and a direct comparison between $\Gamma_{o}$ for FSRQs and HBLs/EHBLs is not possible.
}

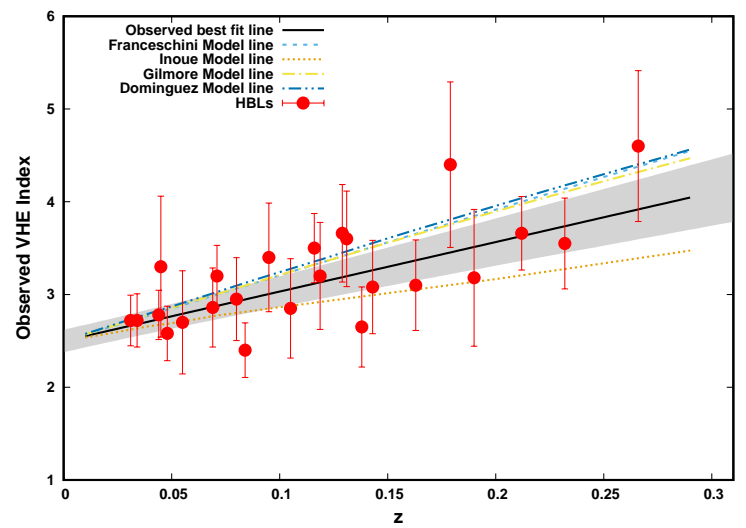

Figure 5. Comparison of observed VHE spectral indices with those predicted by different EBL models for HBLs. Grey region forms the 1- $\sigma$ band on best fit line.

On the contrary, the standard deviation estimated from the whole sample of HBLs considered here is 0.53 which is significantly larger than the variation corresponding to Mkn 421 and Mkn 501. In other words, the range of spectral indices introduced by the EBL induced absorption effect is substantial to over shadow the spectral variation of individual sources. Additionally, the spread of $1-\sigma$ confidence interval of the regression line corresponding to HBLs is 1.1 which is much larger than the spectral variation of individual sources and hence, the associated error on redshift estimation is negligible. This is also evident from the minimal variation in the redshift of the source obtained from the regression lines either using $\Gamma$ or $\Gamma_{a v}$ (Table 2). Nevertheless, for the source PG $1553+113$ the variation in its VHE spectral index during different flux states is observed to be very minimal (Abdo et al. 2010; Abramowski et al. 2015b).

An important conclusion that can be drawn from the present work is the deviation of the predicted VHE spectral indices due to differ- 


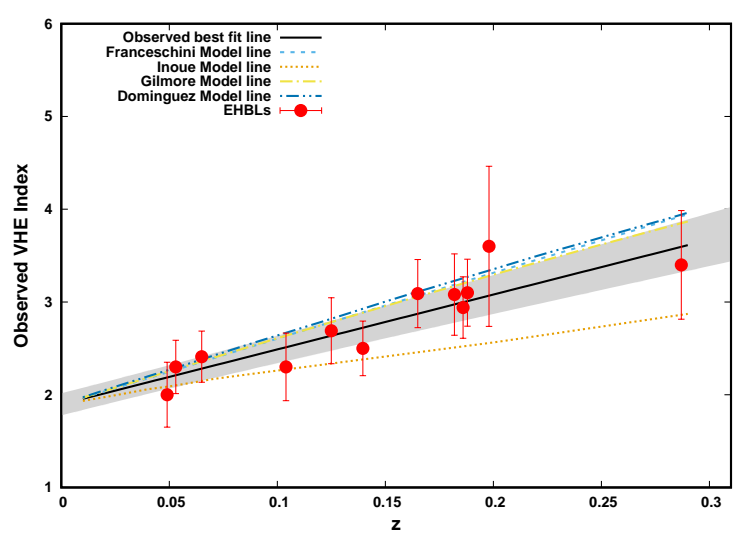

Figure 6. Comparison of observed VHE spectral indices with those predicted by different EBL models for EHBLs. Grey region forms the 1- $\sigma$ band on best fit line.

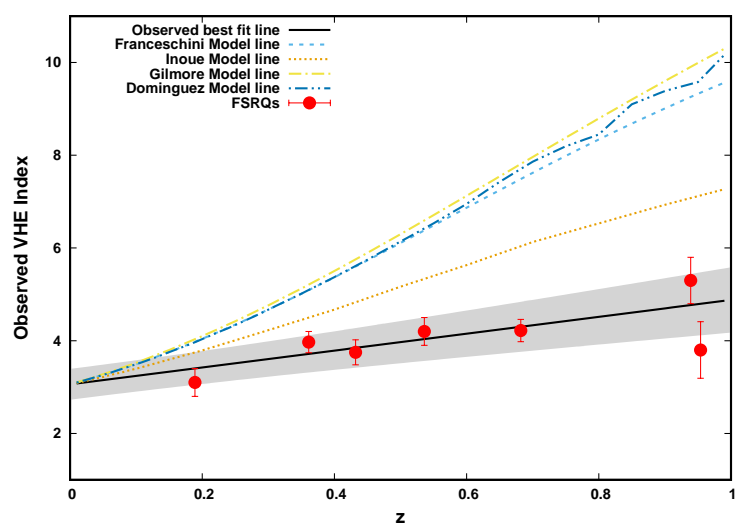

Figure 7. Comparison of observed VHE spectral indices with those predicted by different EBL models for FSRQs. Grey region forms the 1- $\sigma$ band on best fit line.

ent EBL models from the observed trend. At lower redshifts $(0.06 \gtrsim$ $z \lesssim 0.3)$, the EBL estimated through forward evolution model by Inoue et al. (2013) under predicts the spectral indices whereas, the ones estimated from the forward evolution model by Gilmore et al. (2012), backward evolution by Franceschini, A. et al. (2008) and from the observed evolution of galaxy population Domínguez et al. (2010) over predicts in case of HBL/EHBL class. This probably indicate the missing link between assumed cosmological initial conditions with the present epoch. A correction factor can be introduced as a function of $(z)$ in these EBL models to make them consistent with the regression line. However, this should be scrutinized thoroughly using the acceptable cosmological models and the quantities derived from observations. In case of FSRQs, which can probe high redshifts $(z \gtrsim 0.3)$, all the EBL models over predicts the VHE spectral indices. However, the target energy at which the spectral index is calculated for the case of FSRQs is different from HBL/EHBL class and hence they probe different energy regimes of EBL (section §4). It is interesting to note that the EBL model by Inoue et al. (2013) under predicts the observed index in case of BL Lacs while it over predicts for the case of FSRQs. Again this can be due to the difference in the EBL energy probed by these studies. Nevertheless, this study highlights the severe discrepancy between the observations and models. A deeper investigation into this discrepancy involves detailed modelling of cosmological evolution which is beyond the scope of present work.
Most of techniques used to estimate the redshift of blazars through VHE spectral study were based on the prediction of intrinsic source VHE spectrum. It is often straight forward to extrapolate the high energy spectrum to VHE energies and consider it as the upper limit for the intrinsic VHE spectrum. Such studies are capable of providing strong upper limits on the redshifts of various blazars (Archer et al. 2018; Acciari et al. 2010a). Alternatively, one can consider the emission from the hardest particle distribution that can be obtained through Fermi acceleration process as an approximation of intrinsic VHE spectrum (Aharonian et al. 2006a). This in comparison with the observed VHE spectrum can be used to estimate the redshift under certain EBL model (Mazin \& Goebel 2007). The intrinsic VHE spectrum can also be obtained through broadband SED modelling using synchrotron and SSC emission processes (Mankuzhiyil et al. 2010). This knowledge can be used to identify the redshift of unknown blazars from their observed VHE spectrum and an appropriate EBL model. This approach of redshift estimation has an additional advantage that the intrinsic VHE spectrum is estimated from the broadband spectral information rather than a narrow window at high energies. Prandini et al. (2010) introduced a different approach to estimate the redshift of blazars by extrapolating of high energy spectrum of blazars with known redshifts $(z)$ to VHE energies. Assuming this as the intrinsic VHE spectrum, they estimated the redshift $\left(z^{*}\right)$ using the different EBL models. Using a linear regression analysis between $z$ and $z^{*}$, they were able to predict the redshift of blazars which was not known. However, these techniques have a bias on various assumptions of the intrinsic VHE spectrum and/or the EBL models. The present study, on the contrary, do not have such bias and the redshift estimates can be treated as model independent.

A major assumption in the present approach of redshift estimation is that the intrinsic VHE spectral indices of different classes of blazars are similar. At VHE energies the inverse Compton emission happens mostly at Klein-Nishina regime and the spectral index depends on the emitting electron energies, target photon frequency and the bulk Lorentz factor of the jet (Tavecchio et al. 1998). Hence, this assumption demands that these physical quantities do not vary significantly for a particular class of blazars and the shape/slope of their underlying electron distribution responsible for VHE emission is also similar. The shape/slope of the emitting electron distribution is decided by the acceleration rate and the particle diffusion from the main acceleration region (Kirk et al. 1998; Rieger et al. 2007) and this assumption also implies the particle dynamics in blazar jets are comparable. An alternate explanation for the $\Gamma-z$ correlation can be attributed to the cosmological evolution of physical parameters which is particularly effective at VHE energy regime. One such parameter can be the bulk Lorentz factor of the jet which decides the extremity of the Klein-Nishina scattering cross section for the inverse Compton process. However, such studies require rigorous analysis of high quality-simultaneous broadband spectrum of blazars spread over various redshifts. The present approach of redshift estimation can be largely improved with a tighter correlation between the observed VHE spectral index and the redshift. This in turn demands increased number of VHE blazars with precise spectral index measurements. The upcoming high sensitivity experiments such as Cherenkov Telescope Array (CTA)/Major Atmospheric Cherenkov Experiment (MACE) have the potential to achieve this and can be used as an independent tool to estimate the redshift of blazars. 


\section{ACKNOWLEDGEMENT}

Authors thank the anonymous referee for the valuable comments and suggestions. M.Z, S.S, N.I \& A.M acknowledge the financial support provided by Department of Atomic energy (DAE), Board of Research in Nuclear Sciences (BRNS), Govt of India via Sanction Ref No.: 58/14/21/2019-BRNS. SZ is supported by the Department of Science and Technology, Govt. of India, under the INSPIRE Faculty grant (DST/INSPIRE/04/2020/002319). This work has made use of the TeV catalog (http: //tevcat . uchicago . edu/) created and maintained by Scott Wakely and Deirdre Horan and partially supported by NASA and the NSF.

\section{DATA AVAILABILITY}

The data and the codes used in this work will be shared on the reasonable request to the corresponding author Malik Zahoor (email: malikzahoor313@gmail.com).

\section{REFERENCES}

Abdalla H., et al., 2018, Monthly Notices of the Royal Astronomical Society, 482,3011

Abdalla H., et al., 2020b, MNRAS, 494, 5590

Abdalla H., et al., 2020a, Monthly Notices of the Royal Astronomical Society, 494, 5590

Abdo A. A., et al., 2009, ApJ, 707, 1310

Abdo A. A., et al., 2010, ApJ, 708, 1310

Abdollahi S., et al., 2020, The Astrophysical Journal Supplement Series, 247, 33

Abeysekara A. U., et al., 2015, ApJ, 815, L22

Abramowski A., et al., 2011, Astronomy \& Astrophysics, 529, A49

Abramowski A., et al., 2012, Astronomy \& Astrophysics, 542, A94

Abramowski A., et al., 2013a, Monthly Notices of the Royal Astronomical Society 434, 1889-1901

Abramowski A., et al., 2013b, Astronomy \& Astrophysics, 554, A72

Abramowski A., et al., 2013c, Astronomy \& Astrophysics, 559, A136

Abramowski A., et al., 2015b, ApJ, 802, 65

Abramowski A., et al., 2015a, The Astrophysical Journal, 802, 65

Acciari V. A., et al., 2010a, ApJ, 708, L100

Acciari V. A., et al., 2010b, The Astrophysical Journal, 715, L49-L55

Acciari V. A., et al., 2011a, The Astrophysical Journal, 729, 2

Acciari V., et al., 2011b, Astrophys. J., 738, 169

Acciari V. A., et al., 2019, Monthly Notices of the Royal Astronomical Society, 490, 2284-2299

Acciari V. A., Ansoldi S., et al. 2020a,

The Astrophysical Journal Supplement Series

Acciari V. A., et al., 2020b, arXiv e-prints, p. arXiv:2012.11380

Acero F., et al., 2010, Astronomy and Astrophysics, 511, A52

Ackermann M., et al., 2011, The Astrophysical Journal, 743, 171

Ackermann M., et al., 2015, The Astrophysical Journal, 810, 14

Aharonian F., et al., 2006a, Nature, 440, 1018

Aharonian F., et al., 2006b, Astronomy \& Astrophysics, 455, 461-466

Aharonian F., et al., 2007a, Astronomy \& Astrophysics, 470, 475-489

Aharonian F., et al., 2007b, Astronomy \& Astrophysics, 473, L25-L28

Aharonian F., et al., 2007c, Astronomy \& Astrophysics, 475, L9-L13

Aharonian F., et al., 2007d, The Astrophysical Journal, 664, L71

Aharonian F., et al., 2008a, A\&A, 477, 481

Aharonian F., et al., 2008b, Astronomy \& Astrophysics, 481, L103-L107

Aharonian F., et al., 2010, Astronomy and Astrophysics, 521, A69

Ahnen, M. L. et al., 2016, A\&A, 595, A98

Ahnen M. L., et al., 2016a, Monthly Notices of the Royal Astronomical Society, 459,3271

Ahnen M. L., et al., 2016b, A\&A, 595, A98
Ahnen M. L., et al., 2017, Monthly Notices of the Royal Astronomical Society, $468,1534-1541$

Albert J., et al., 2006, THE ASTROPHYSICAL JOURNAL, 648, L105

Albert J., et al., 2007, ApJ, 669, 862

Albert J., et al., 2009, A\&A, 493, 467

Aleksi'c, J. et al., 2012, A\&A, 542, A100

Aleksi'c, J. et al., 2014, A\&A, 567, A135

Aleksi'c, J. et al., 2015, A\&A, 573, A50

Aleksić J., et al., 2011a, ApJ, 729, 115

Aleksić J., et al., 2011b, ApJ, 730, L8

Aleksic J., et al., 2012, Astron. Astrophys., 544, A75

Aleksić J., et al., 2012, Astronomy \& Astrophysics, 539, A118

Aleksić J., et al., 2014, Astronomy \& Astrophysics, 563, A90

Aleksić J., et al., 2015, Monthly Notices of the Royal Astronomical Society, 451, 739-750

Aleksić J., et al., 2016, Astronomy \& Astrophysics, 591, A10

Aliu E., et al., 2011, The Astrophysical Journal, 742, 127

Aliu E., et al., 2012a, The Astrophysical Journal, 750, 94

Aliu E., et al., 2012b, The Astrophysical Journal, 755, 118

Aliu E., et al., 2013, The Astrophysical Journal, 779, 92

Aliu E., et al., 2015, ApJ, 799, 7

Archambault S., et al., 2016, Monthly Notices of the Royal Astronomical Society, 461, 202-208

Archer A., et al., 2018, The Astrophysical Journal, 862, 41

Arlen T., et al., 2012, The Astrophysical Journal, 762, 92

Baloković M., et al., 2016, The Astrophysical Journal, 819, 156

Benbow W., 2011, in International Cosmic Ray Conference. p. 47 (arXiv: 1110.0038), doi:10.7529/ICRC2011/V08/0746

Benbow W., VERITAS Collaboration 2015, in 34th International Cosmic Ray Conference (ICRC2015). p. 821 (arXiv: 1508.07251)

Boller T., Freyberg M. J., Trümper J., Haberl F., Voges W., Nandra K., 2016, Astronomy \& Astrophysics, 588, A103

Brissenden R. J. V., Remillard R. A., Tuohy I. R., Schwartz D. A., Hertz P. L., 1990, ApJ, 350, 578

Cerruti M., 2011, in International Cosmic Ray Conference. p. 109 (arXiv: 1110.2119), doi:10.7529/ICRC2011/V08/0913

Costamante L., Ghisellini G., 2002, A\&A, 384, 56

Danforth C. W., Keeney B. A., Stocke J. T., Shull J. M., Yao Y., 2010, The Astrophysical Journal, 720, 976

Dermer C. D., Schlickeiser R., 1993, ApJ, 416, 458

Djannati-Ataï, A. et al., 2002, A\&A, 391, L25

Domínguez A., et al., 2010, Monthly Notices of the Royal Astronomical Society, 410, 2556-2578

Donato, D. Ghisellini, G. Tagliaferri, G. Fossati, G. 2001, A\&A, 375, 739

Donato D., Sambruna R., Gliozzi M., 2005, Astronomy and Astrophysics, 433

Dwek E., Krennrich F., 2013, Astroparticle Physics, 43, 112

Falomo R., Bersanelli M., Bouchet P., Tanzi E. G., 1993, AJ, 106, 11

Falomo R., Scarpa R., Bersanelli M., 1994, ApJS, 93, 125

Farina E. P., Falomo R., Decarli R., Treves A., Kotilainen J. K., 2012, Monthly Notices of the Royal Astronomical Society, 429, 1267

Finke J. D., Razzaque S., Dermer C. D., 2010, The Astrophysical Journal, 712,238

Foffano L., Prandini E., Franceschini A., Paiano S., 2019, Monthly Notices of the Royal Astronomical Society, 486, 1741

Fortin P., Aharonian F. A., Hofmann W., Rieger F., 2008, AIP Conference Proceedings

Franceschini A., Rodighiero G., 2017, Astronomy \& Astrophysics, 603, A34

Franceschini, A. Rodighiero, G. Vaccari, M. 2008, A\&A, 487, 837

Furniss A., et al., 2013, ApJ, 768, L31

Gaidos, J. A. 1996, Nature, 383

Gaté F., H. E. S. S. Collaboration Fitoussi T., 2017, in 35th International Cosmic Ray Conference (ICRC2017). p. 645 (arXiv: 1708. 09612)

Gilmore R. C., Somerville R. S., Primack J. R., Domínguez A., 2012, Monthly Notices of the Royal Astronomical Society, 422, 3189

Ginzburg V. L., Syrovatskii S. I., 1965, ARA\&A, 3, 297

Gould R. J., Schréder G. P., 1967, Physical Review, 155, 1404 
Griffiths R. E., Wilson A. S., Ward M. J., Tapia S., Ulvestad J. S., 1989, MNRAS, 240, 33

H. E. S. S. Collaboration et al., 2019, A\&A, 627, A159

H. E. S. S. Collaboration et al., 2020, A\&A, 633, A162

Hauser M. G., Dwek E., 2001, Annual Review of Astronomy and Astrophysics, 39,249

Inoue Y., Inoue S., Kobayashi M. A. R., Makiya R., Niino Y., Totani T., 2013, The Astrophysical Journal, 768, 197

Jones D. H., et al., 2004, Monthly Notices of the Royal Astronomical Society, $355,747-763$

Jones D. H., et al., 2009a, Monthly Notices of the Royal Astronomical Society, 399, 683

Jones D. H., et al., 2009b, Monthly Notices of the Royal Astronomical Society, 399, 683-698

Khaire V., Srianand R., 2015, The Astrophysical Journal, 805, 33

Kirk J. G., Rieger F. M., Mastichiadis A., 1998, A\&A, 333, 452

Kneiske, T. M. Dole, H. 2010, A\&A, 515, A19

Kneiske T. M., Bretz T., Mannheim K., Hartmann D. H., 2004, A\&A, 413, 807

Landoni, M. Falomo, R. Treves, A. Sbarufatti, B. 2014, A\&A, 570, A126

Laurent-Muehleisen S. A., Kollgaard R. I., Feigelson E. D., Brinkmann W., Siebert J., 1999, ApJ, 525, 127

MAGIC Collaboration et al., 2018, A\&A, 619, A159

MAGIC Collaboration: et al., 2020, A\&A, 637, A86

Mankuzhiyil N., Persic M., Tavecchio F., 2010, The Astrophysical Journal, 715, L16

Mazin D., Goebel F., 2007, ApJ, 655, L13

Mücke A., Protheroe R. J., Engel R., Rachen J. P., Stanev T., 2003, Astroparticle Physics, 18, 593

Oh K., et al., 2018, The Astrophysical Journal Supplement Series, 235, 4

Pei Y. C., Fall S. M., Hauser M. G., 1999, ApJ, 522, 604

Peter, D. Domainko, W. Sanchez, D. A. van der Wel, A. Gässler, W. 2014, A\&A, 571, A41

Prandini E., Bonnoli G., Maraschi L., Mariotti M., Tavecchio F., 2010, Monthly Notices of the Royal Astronomical Society: Letters, 405, L76

Prandini E., Bonnoli G., Maraschi L., Mariotti M., Tavecchio F., 2011, arXiv e-prints, p. arXiv:1101.4098

Primack J. R., 2005, AIP Conference Proceedings

Prokoph H., Becherini Y., Böttcher M., Boisson C., Lenain J. P., Sushch I., 2015, H.E.S.S. discovery of very-high-energy gamma-ray emission of PKS 1440-389 (arXiv: 1509.03972)

Ratcliffe A., Shanks T., Broadbent A., Parker Q. A., Watson F. G., Oates A. P., Fong R., Collins C. A., 1996, Monthly Notices of the Royal Astronomical Society, 281, L47

Rau, A. et al., 2012, A\&A, 538, A26

Rieger F. M., Bosch-Ramon V., Duffy P., 2007, Astrophysics and Space Science, 309, 119-125

Sahu S., López Fortín C. E., Nagataki S., 2019, ApJ, 884, L17

Sanchez, D. A. Fegan, S. Giebels, B. 2013, A\&A, 554, A75

Sbarufatti B., Treves A., Falomo R., 2005, The Astrophysical Journal, 635, 173

Sbarufatti B., Treves A., Falomo R., Heidt J., Kotilainen J., Scarpa R., 2006, AJ, 132, 1

Shaw M. S., et al., 2013, The Astrophysical Journal, 764, 135

Shectman S. A., Landy S. D., Oemler A., Tucker D. L., Lin H., Kirshner R. P., Schechter P. L., 1996, ApJ, 470, 172

Sinha A., Sahayanathan S., Misra R., Godambe S., Acharya B. S., 2014, The Astrophysical Journal, 795, 91

Stecker F. W., Malkan M. A., Scully S. T., 2006, The Astrophysical Journal, 648, 774

Tagliaferri G., et al., 2008, The Astrophysical Journal, 679, 1029

Tanaka Y. T., et al., 2014, ApJ, 787, 155

Tavecchio F., 2014, MNRAS, 438, 3255

Tavecchio F., Maraschi L., Ghisellini G., 1998, ApJ, 509, 608

Treves A., Falomo R., Uslenghi M., 2007, A\&A, 473, L17

Urry C. M., Padovani P., 1995, PASP, 107, 803

Vassiliev V. V., 2000, Astroparticle Physics, 12, 217

Veron-Cetty M. P., Veron P., 1993, A\&AS, 100, 521

Vettolani, G. et al., 1998, Astron. Astrophys. Suppl. Ser., 130, 323
Weidinger M., Spanier F., 2015, A\&A, 573, A7

This paper has been typeset from a $\mathrm{T}_{\mathrm{E}} \mathrm{X} / \mathrm{L} \mathrm{T}_{\mathrm{E}} \mathrm{X}$ file prepared by the author. 